\title{
Advanced Conduction Angle Control of Permanent Magnet Brushless Motor Drives
}

\author{
Jinyun Gan, C.C. Chan, K. T. Chau, and J. Z. Jiang \\ Department of Electrical and Electronic Engineering \\ The University of Hong Kong \\ Pokfulam Road \\ Hong Kong
}

\begin{abstract}
A novel advanced conduction angle control scheme for permanent magnet brushless motor drives is presented in this paper. The originality of this scheme is to employ the transformer EMF in stator windings to counteract the rotational EMF by controlling the advanced conduction angle when the motor drive operates above the base speed. Hence the constant-power operation region can be extended considerably, even though the current regulator is in saturation. The attractive feature of the proposed control scheme is that it has better controllability and easier to implement than conventional flux-weakening control scheme because of the absence of coordinate transformation. It can be applied to all PM brushless motors, including conventional, phase-decoupling, sinusoidal-fed and rectangular-fed types. An Intel 8098-based digital controller and a phase-decoupling rectangular-fed PM brushless motor are employed to implement the scheme. The hardware and the software are simple and flexible. Both computer simulation and experimental results of a $1-\mathrm{kW}$ motor drive system verifies that the proposed control scheme works well.
\end{abstract}

\section{Introduction}

Permanent magnet (PM) brushless motors are attracting growing attention for a wide variety of industrial applications [1]. Because of currentfree PM excitation, as well as elimination of brushes and slip rings; the PM brushless motors have higher efficiency, higher power density, simpler structure and better controllability than conventional motors [2]. These characteristics are particularly attractive for adjustable-speed motor drive applications such as electric vehicle (EV) applications.

In most applications, particularly for EV drives, both constant torque operation below the base speed and constant power operation above the base speed are necessary. The constant torque operation can be readily achieved, whereas the constant power operation can not be directly achieved due to fixed field excitation provided by PM. For overcoming this limitation, new PM motor structure design [3] and flux weakening control algorithms [4], [5] have been proposed to achieve flux weakening operation similar to field weakening operation in a conventional dc motor. However, the proposed control algorithm is only directly applicable to sinusoidal-fed PM motors, because it needs coordinate transformation between $a-b-c$ phase variables and $d-q$ variables. For rectangular-fed PM motors, it is not readily applicable. Although Fourier analysis can be used to express the rectangular wave into the corresponding fundamental and harmonic sinewaves, it is too cumbersome to be realized. Furthermore when the motor is phase-decoupling such that the mutual inductance between phase windings is negligible, the proposed control algorithm is ill-suited [7].

The purpose of this paper is to use a novel control scheme to provide wide speed range operation for the phase-decoupling rectangularfed PM brushless motor by means of the transformer EMF component in stator windings, providing an equivalent flux weakening effect when the speed exceeds the base speed. The proposed control scheme can be applied to all different types of PM brushless motors [8]. An Intel 8098 microprocessor will be used to implement this control scheme. The structure of hardware and software will be presented in this paper. Both computer simulation and experimental verification of torque-speed performance during constant power operation will also be given. 


\section{Phase-Decoupling PM Brushless Motor Drive}

As shown in Fig. 1, the schematic diagram of a novel 5-phase 22-pole phase-decoupling rectangular-fed PM brushless motor drive is used for exemplification. It consists of two parts: motor and inverter. A suitable control strategy should be applied to make it running properly. The principle of operation has been described in [6], [7]. Here, a brief description is given.

\subsection{Overview of Motor}

The five phase voltage waveform is shown in Fig. 2. As shown, at any instant, there are four phase windings in the conducting state. Each phase winding conducts for $144^{\circ}$ electrical degree, then remains non-conducting state for $36^{\circ}$ in each half cycle. The phase shift between adjacent phase is $36^{\circ}$. To ensure that each winding current produces positive torque, the conducting state should be decided by rotor position signals which are generated by a shaft sensor. Five-phase position signals are shown in Fig. 3, which are sent out by five optodevices.

The attractive features of the motor are high power density, high efficiency and excellent controllability. Besides these, the flux path of each phase is independent, namely the mutual inductance between phase windings is negligible, or simply phase-decoupling [6]-[9]. Therefore, each phase winding has a similar model like a conventional dc motor. The voltage equation of the $\mathrm{j}$-th phase can be expressed as:

$$
\begin{aligned}
v_{j}\left(\omega t+\theta_{0}-\frac{(j-1) \pi}{5}\right) & =R i_{j}\left(\omega t+\theta_{0}-\frac{(j-1) \pi}{5}\right) \\
+ & L \frac{d i_{j}}{d t}\left(\omega t+\theta_{0}-\frac{(j-1) \pi}{5}\right) \\
& +e_{j}\left(\omega t-\frac{(j-1) \pi}{5}\right)
\end{aligned}
$$

where $j=1 \sim 5, v_{j}$ is the applied phase voltage, $R i_{j}$ is the resistance voltage drop, $L \frac{d i_{j}}{d t}$ is the transformer EMF due to the self-inductance, $e_{j}$ is the rotational EMF induced by the rotating PM field and is proportional to the motor speed, $\theta_{0}$ is the advanced conduction angle (namely the shifted angle of the applied phase voltage leading ahead the rotational EMF of the corresponding phase).

The electromagnetic torque and motion equations are given by:

$$
\begin{aligned}
T_{e}(\omega t)= & \frac{1}{\omega} \sum_{j=1}^{5} e_{j}\left(\omega t-\frac{(j-1) \pi}{5}\right) \\
& \times i_{j}\left(\omega t+\theta_{0}-\frac{(j-1) \pi}{5}\right) \\
T_{e}(\omega t)- & T_{l}-B \omega(t)=J \frac{d \omega(t)}{d t}
\end{aligned}
$$

where $T_{e}$ is the electromagnetic torque, $T_{l}$ is the load torque, $\omega$ is the angular speed, $B$ is the damping coefficient, and $J$ is the moment of inertia. Equations (1)-(3) combined with motor parameters and conducting state can be used to simulate the dynamic performance of the motor.

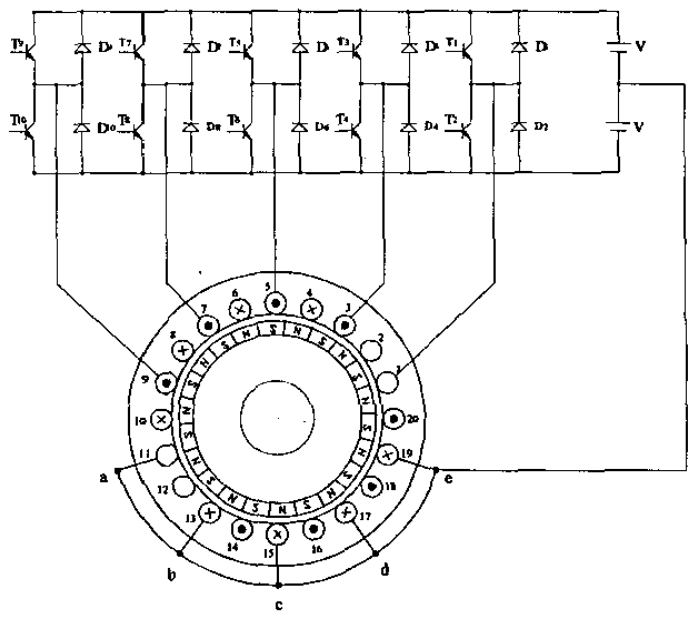

Fig. 1. Phase-decoupling rectangular-fed PM motor drive

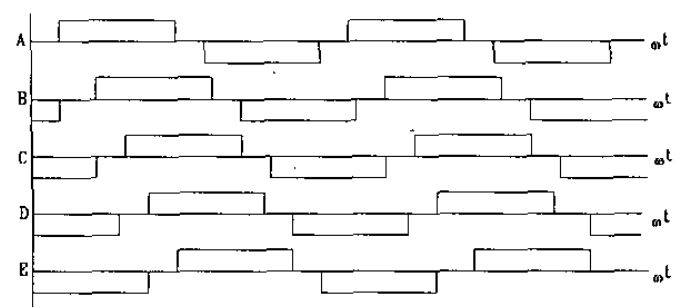

Fig. 2. Five-phase voltage waveforms 


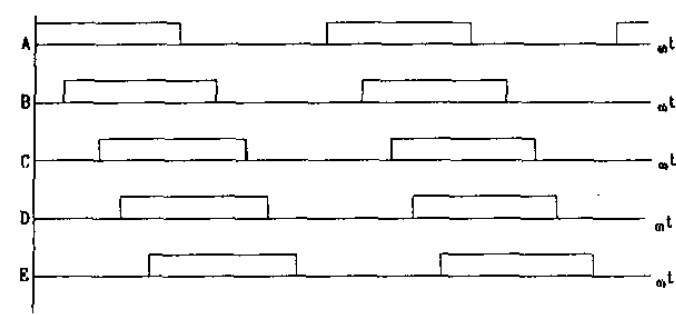

Fig. 3. Five-phase position signals

\subsection{Novel Control Scheme}

When the motor speed is lower than the base speed, the applied voltage is always larger than the rotational EMF, so the current regulator is fully operational. The hysteresis current control is applicable for constant torque operation. And the results are satisfactory. In this case, the advanced conduction angle $\theta_{0}$ is fixed and commonly set to zero, so that the phase current always produces positive torque in conduction period.

As the speed is increased above the base speed, the rotational EMF rises up to near the applied voltage, resulting in rapidly reduction in the phase current and the electromagnetic torque. The current regulator is saturated and loses control ability to follow the reference current. The maximum operation speed is limited in a lower level. Conventionally, the flux-weakening control is used to extend constant power operation regime for sinusoidal-fed PM brushless motor drives. It is ill-suited for the proposed motor drive because of its rectangular-fed and phase-decoupling features. Therefore a novel control scheme is desired to improve its torquespeed performance at high speeds.

Further investigation shows that the above problem can be solved if the advanced conduction angle $\theta_{0}$ is variable. The novel control scheme is to purposely employ the transformer EMF to counteract the rotational EMF which is even larger than the applied voltage at high speeds. This transformer EMF, proportional to the derivative of phase current, is controlled by $\theta_{0}$. The simulation result is shown in Fig. 4. When $\theta_{0}$ has a suitable positive value, at the beginning of conduction with lower rotational EMF, the phase current quickly rises up and the transformer EMF is positive which indicates that energy is stored in the phase winding. The phase current reaches the maximum value when the rotational EMF equals the applied voltage. After that, the rotational EMF is larger than the applied voltage. The phase winding begins to release energy and the phase current decreases gradually. Since the corresponding transformer EMF is negative, it assists the applied voltage to counteract the rotational EMF. By varying the advanced conduction angle, the phase current waveform can be shaped, hence the phase current is regulated. In this way, an equivalent flux weakening effect can be taken to achieve the constant power operation even though the current regulator is in saturation. This control scheme is suitable to all PM brushless motor drives, including sinusoidal-fed, rectangular-fed or phase-decoupling types.

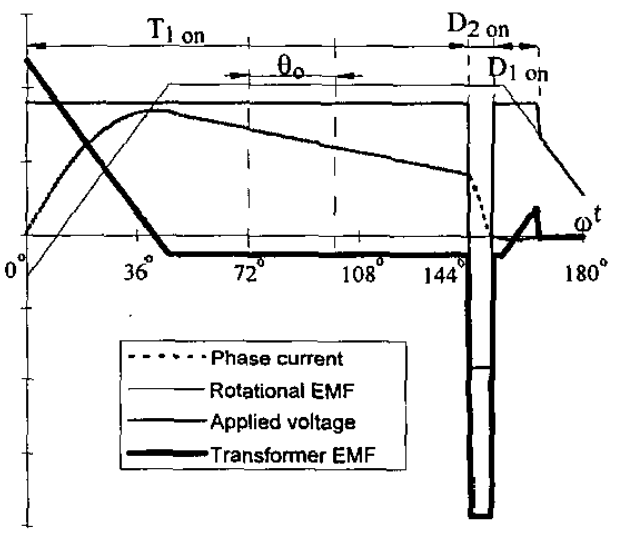

Fig. 4. Waveforms of advanced conduction

\section{Control System}

A microprocessor-based control system has been set up for verifying the proposed control scheme. The dual closed-loop control of the speed and current is adopted. As the motor is phasedecoupling, each phase current is controlled independently without using motor parameters and coordinate transformation. The system is simple, as shown in Fig. 5. 


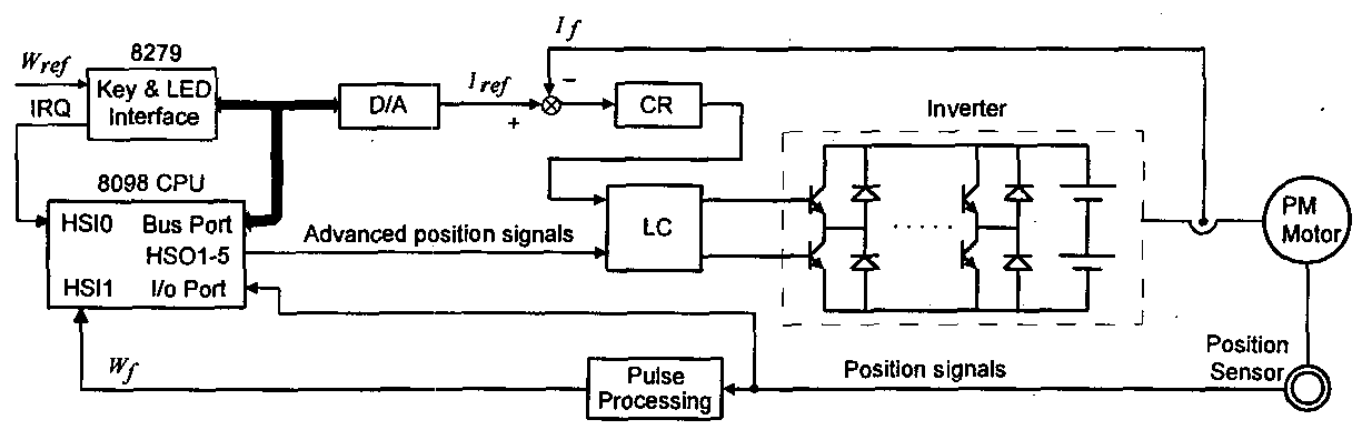

Fig. 5. Control system block diagram

\subsection{Hardware Structure}

An Intel 8098 16-bit microcontroller is the core of the drive control system. It combines with the position sensor and pulse processing circuit to form the speed regulator loop. Five phase position signals from optodevices are summed up to a serial speed feedback signal by the pulse processing circuit. This signal is sent to 8098 CPU through HSI1 port and is used to measure the speed of motor. The speed reference is inputted by a mini keyboard. The error of speed is used to deduce the magnitude of current reference by a digital PI regulator implemented by software. A D/A circuit transfers the digital current reference into analogue for the current loop. An inverter with the dc link voltage $172 \mathrm{~V}$ is used to feed the windings of motor. Hall-effect current sensors provide phase current feedback to close the phase current regulator (CR) loop using the hysteresis-band PWM principle. The output of $C R$ and the position signals with advanced conduction angle from HSO1-HSO5 pins are combined by a logic circuit (LC) to form the firing pulse signal for the inverter. An 8279 chip is used as the interface between CPU and auxiliary elements of keyboard and LED display.

\subsection{Software Implementation}

Among the 8098 high-speed I/O units, a 16-bit timer (T1), a flexible 16-bit counter (T2) and programmable high-speed input (HSI) / output (HSO) units take the main task of the control scheme. Based on these powerful units, the software is very simple and flexible. Besides the main program used for initialising the system, three interrupt service subprograms (INT-SUB) are necessary. One is T1 software timer INTSUB. The sampling interval of the speed signal is set by it. Speed calculation and digital PI regulation are also arranged in it. In order to save memory space, an incremental mode of PI algorithm is adopted. Another one is HSI INTSUB. The HSI unit has the function of recording the value of timer $\mathrm{Tl}$ at the moment of pulse tripping. So the corresponding HSI INT-SUB is set to take measurement of the pulse number and their duration period for speed measurement. The last one is HSO INT-SUB. The HSO unit has six output pins. Each can send out a desired waveform through command settings. And the change of the pin level causes an interruption request. The main objective of HSO INT-SUB is to set the HSO-command properly, so that HSO1-HSO5 pins output 5-phase rotor position pulses like Fig. 3. When the speed is lower than the base speed, the commands based on $\mathrm{T} 2$ counter counting pulses from HSIl pin enable the output position pulses to follow the signals from the position sensor. As the speed above the base speed, according to the time value recorded by the HSI unit, the rotor position angle can be converted into an equivalent time value. And the command based on $\mathrm{T} 1$ timer enables the output position pulse with an advanced conduction angle for constant power operation. The magnitude of the advanced angle $\theta_{0}$ can thus be easily set.

\section{Experimental Results}

An 1-kW motor drive system is used for experimental tests. Its parameters are listed in the 
Appendix. When it runs below the base speed of $500 \mathrm{r} / \mathrm{min}, \theta_{0}$ is held at zero to develop maximum torque/ampere, and the hysteresis current regulator takes effect to keep constant torque operation. The measured current waveform at the base speed is shown in Fig. 6(a). It is clear that the current regulator works well. Whereas, when it runs above the base speed, the advanced conduction angle control is used for constant power operation. The higher the speed is, the bigger is $\theta_{0}$. Because of the negative torque component existed in the advanced conduction period, an excessive $\theta_{0}$ is harmful to the system performance at a given operating point. An optimal value of $\theta_{0}$ can be achieved by simulation. In general, a minimum $\theta_{0}$ satisfying speed and torque needs is a reasonable choice. With the advanced conduction angle of $53^{\circ}$, the current waveform of constant power operation at the triple base speed of $1500 \mathrm{r} / \mathrm{min}$ is shown in Fig. 6(b). It is consistent with the waveform shown in Fig. 4.

For further verifying the effect of the novel control scheme, experimental torque-speed curves with different $\theta_{0}$ are given in Fig. 7 . These curves indicate that the torque-speed can be extended considerably by increasing $\theta_{0}$. It should be noted that these curves are the natural characteristics of the motor drive, namely the experiment is done under open-loop operation. While under closed-loop operation, $\theta_{0}$ is not unique for each operating point. The minimum value of $\theta_{0}$ can be directly achieved from these curves.

By increasing the advanced conduction angle adequately, the experimental motor drive can operate in constant power region. Fig. 8 is the measured torque-speed curve in constant power operation. The minimum value of $\theta_{0}$ is also marked near the corresponding point. It can be seen that with $\theta_{0}$ increasing from $0^{\circ}$ to $60^{\circ}$, the proposed motor drive can successfully achieve constant-power operation up to $2250 \mathrm{r} / \mathrm{min}$, more than four times the base speed. Furthermore, without employing the proposed control scheme, it can be found that the corresponding torque- speed curve (dotted line) has a narrow operating range above the base speed.

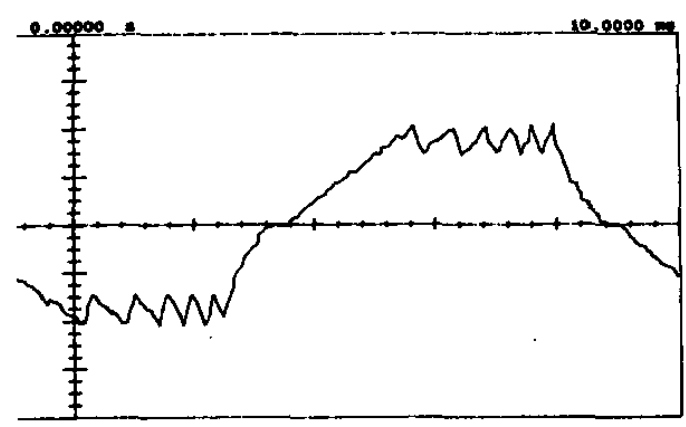

(a) Constant torque operation at $500 \mathrm{r} / \mathrm{min}$ (5A/div, 2ms/div)

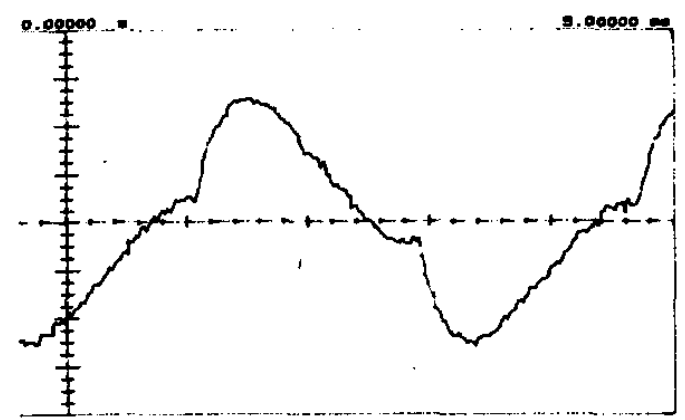

(b) Constant power operation at $1500 \mathrm{r} / \mathrm{min}$ (2.A/div, $1 \mathrm{~ms} / \mathrm{div})$

Fig. 6. Measured current waveforms

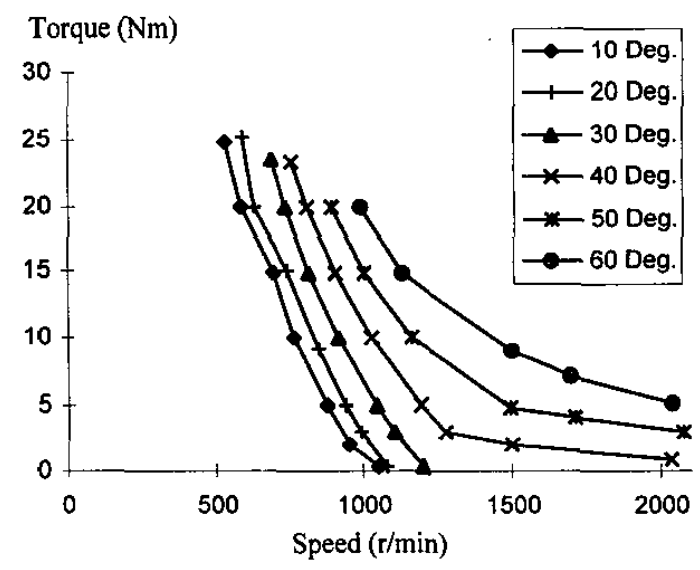

Fig. 7. Measured torque-speed characteristics as increasing $\theta_{\text {。 }}$ 


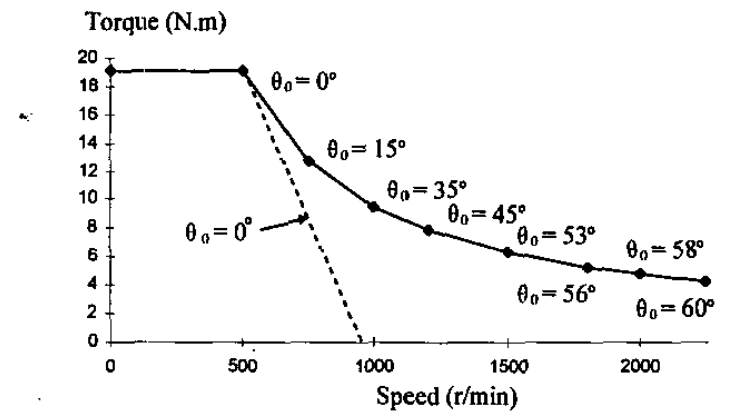

Fig. 8. Torque-speed curve in constant power operation

\section{Conclusion}

A microcontroller-based phase-decoupling rectangular-fed PM brushless motor drive system has been presented which operates successfully in both constant torque and constant power regions. Due to the phase-decoupling feature, a novel control scheme is presented for constant power operation, which fits for all kinds of PM brushless motor. The originality of this scheme is to employ the transformer EMF to counteract the rotational EMF by controlling the advanced conduction angle when the motor operates above the base speed. The speed range can thus be extended considerably. A 5-phase 22-pole motor drive is used for exemplification, and operates up to more than four times the base speed. The simulation and experimental results have verified the validity of the proposed advanced conduction angle control for wide speed range operation.

\section{Appendix - Motor Parameters}

$\begin{array}{ll}\text { Rated power } & 1.0 \mathrm{~kW} \\ \text { Rated voltage } & 2 \times 86 \mathrm{~V} \\ \text { Rated phase current } & 7.5 \mathrm{~A} \\ \text { Base Speed } & 500 \mathrm{r} / \mathrm{min} \\ \text { Number of phases } & 5 \\ \text { Number of poles } & 22 \\ \text { Number of slots } & 20 \\ \text { Number of coils } & 10 \\ \text { Winding type } & \text { Single layer } \\ \text { Slot pitch } & 1 \\ \text { Resistance per phase }\left(75^{\circ}\right) & 0.306 \Omega \\ \text { Self-inductance per phase } & 6.735 \mathrm{mH} \\ \text { Magnet material } & \mathrm{Nd}-\mathrm{Fe}-\mathrm{B}\end{array}$

\section{References}

[1] T. M. Jahns, "Motion control with permanent magnet ac machines," Proc. IEEE, vol. 82, 1994.

[2] T. J. E. Miller, Brushless Permanent Magnet And Reluctance Motor Drives. Oxford University Press, New York, 1989.

[3] L. Xu, L. Ye, L. Zhen and A. El-Antably, "A new design concept of permanent magnet machine for flux weakening operation," IEEE Trans. IA., vol. 31, pp.373-376, 1995.

[4] - B. Sneyers, D.W. Novotny, and T. A. Lipo, "Field weakening in buried permanent magnet AC motor drives," IEEE Trans. IA, vol. 21, pp. 398-407, 1985.

[5] T. M. Jahns, "Flux-weakening regime operation of an interior permanent magnet synchronous motor drive," IEEE Trans. IA, vol. 23 pp. 681-689, 1987.

[6] C. C. Chan, "A novel permanent magnet brushless DC motor," UK Application, 1994.

[7] C. C. Chan, J. Z. Jiang, G. H. Chen, X. Y. Wang and K. T. Chau, "A novel polyphase multipole squarewave permanent magnet motor drive for electric vehicles," IEEE Trans. IA, vol. 30, pp. 1258-1266, 1994.

[8] C. C. Chan, J. Z. Jiang, W. Xia and K. T. Chau, "Novel wide range speed control of permanent magnet brushless motor drive," IEEE Trans. PE, vol. 10, pp. 539-546, 1995.

[9] P. J. McCleer, J. M. Bailey, J. S. Lawler, and B. Banerjee, "Five phase trapezoidal back emf PM synchronous machines and drives," Proc. EPE Conf., Italy, 1990. 